OPEN ACCESS

Edited by:

Kai Zhou,

Zhejiang University, China

Reviewed by:

Pedro Xavier-Elsas,

Federal University of Rio de

Janeiro, Brazl

Yvonne Pfeifer,

Robert Koch Institute, Germany

Richard Vernon Goering,

Creighton University, United States

${ }^{*}$ Correspondence:

Trevor Lithgow

trevor.lithgow@monash.edu

Tieli Zhou

wyztli@163.com

Jianming Cao

wzcjming@163.com

Specialty section:

This article was submitted to Infectious Diseases - Surveillance

Prevention and Treatment,

a section of the journal

Frontiers in Public Health

Received: 28 March 2019

Accepted: 30 July 2019

Published: 19 August 2019

Citation:

Zhao Y, Zhang X, Torres WL, Liu H, Rocker A, Zhang Y, Wang J, Chen L,

Bi W, Lin J, Strugnell RA, Zhang S,

Lithgow T, Zhou T and Cao J (2019)

An Outbreak of

Carbapenem-Resistant and

Hypervirulent Klebsiella pneumoniae in

an Intensive Care Unit of a Major

Teaching Hospital in Wenzhou, China.

Front. Public Health 7:229.

doi: 10.3389/fpubh.2019.00229

\section{An Outbreak of}

\section{Carbapenem-Resistant and} Hypervirulent Klebsiella pneumoniae in an Intensive Care Unit of a Major Teaching Hospital in Wenzhou, China

\author{
Yajie Zhao ${ }^{1}$, Xiucai Zhang ${ }^{2}$, Von Vergel L. Torres ${ }^{3}$, Haiyang Liu ${ }^{2}$, Andrea Rocker ${ }^{3}$, \\ Yizhi Zhang ${ }^{2}$, Jiawei Wang ${ }^{3}$, Lijiang Chen ${ }^{2}$, Wenzi Bi ${ }^{4}$, Jie Lin ${ }^{2}$, Richard A. Strugnell ${ }^{5}$, \\ Siqin Zhang ${ }^{2}$, Trevor Lithgow ${ }^{3 *}$, Tieli Zhou ${ }^{2 *}$ and Jianming Cao ${ }^{1 *}$
}

' School of Laboratory Medicine and Life Science, Wenzhou Medical University, Wenzhou, China, ${ }^{2}$ Department of Clinical Laboratory, The First Affiliated Hospital of Wenzhou Medical University, Wenzhou, China, ${ }^{3}$ Infection and Immunity Program, Department of Microbiology, Biomedicine Discovery Institute, Monash University, Melbourne, VIC, Australia, ${ }^{4}$ School of Medicine, The Fourth Affiliated Hospital of Zhejiang University, Jinhua, China, ${ }^{5}$ Department of Microbiology and Immunology, The Peter Doherty Institute, The University of Melbourne, Melbourne, VIC, Australia

Carbapenem-resistant, hypervirulent Klebsiella pneumoniae (CR-hvKP) has recently emerged as a significant threat to public health. In this study, 29 K. pneumoniae isolates were isolated from eight patients admitted to the intensive care unit (ICU) of a comprehensive teaching hospital located in China from March 2017 to January 2018. Clinical information of patients was the basis for the further analyses of the isolates including antimicrobial susceptibility tests, identification of antibiotic resistance and virulence gene determinants, multilocus sequence typing (MLST), Xbal-macrorestriction by pulsed-field gel electrophoresis (PFGE). Selected isolates representing distinct resistance profiles and virulence phenotypes were screened for hypervirulence in a Galleria mellonella larvae infection model. In the course of the outbreak, the overall mortality rate of patients was $100 \%(n=8)$ attributed to complications arising from CR-hvKP infections. All isolates except one (28/29, 96.6\%) were resistant to multiple antimicrobial agents, and harbored diverse resistance determinants that included the globally prevalent carbapenemase blakPC-2. Most isolates had hypervirulent genotypes being positive for 19 virulence-associated genes, including iutA (25/29, 86.2\%), rmpA (27/29, 93.1\%), ybtA (27/29, 93.1\%), entB (29/29, 100\%), fimH (29/29, 100\%), and $m r k D$ (29/29, 100\%). MLST revealed ST11 for the majority of isolates (26/29, 89,7\%). Infection assays demonstrated high mortality in the Galleria mellonella model with the highest LD 50 values for three isolates ( $<10^{5} \mathrm{CFU} / \mathrm{mL}$ ) demonstrating the degree of hypervirulence of these CR-hvKP isolates, and is discussed relative to previous outbreaks of CR-hvKP.

Keywords: Klebsiella pneumoniae, hypervirulent, carbapenem-resistance, ST11, epidemiology 


\section{INTRODUCTION}

Klebsiella pneumoniae is a Gram-negative pathogen causing hospital-acquired infections, especially for immunocompromised patients in the intensive care units (ICU) (1-3). Variants called hypervirulent K. pneumoniae (hvKp) have evolved (4) which are becoming more prevalent. Diagnostically, hvKp tend to be hypermucoviscous due to increased secretion of capsular polysaccharide, and metabolically more robust through the acquisition of genes for more efficient iron acquisition from host tissues (2). Isolates of hvKP usually belong to sequence type ST23 and their capsule is usually of the fucosylated K1-type (5). There is also mounting evidence that hvKp secrete factors which impact the epithelia and microbiome, such as the cell-cycle modulating non-ribosomal peptide colibactin and the channel-forming bacteriocin microcin E492 (6, 7), respectively. These factors modify the host niche and might thereby contribute to the observed increase in virulence $(6,8)$. The significance of these features has been established in the measurement of virulence phenotype in vitro and in vivo (9-11), and whole genome sequence analyses document the range of genes for iron-acquisition, enhanced capsule secretion, and toxin secretion. These include iron-binding siderophores: aerobactin, yersiniabactin, and salmochelin, as well as TonBdependent transporters for iron $(\mathrm{Kfu})$, iron dicitrate $(\mathrm{Fec})$, and ferric siderophores (FyuA) $(7,12)$.

Carbapenem-resistant $K$. pneumoniae (CRKP) are another global threat given that until recently carbapenems were last line drugs for Klebsiella infections (13-15). Carriage of the gene $b l a_{\mathrm{KPC}-2}$ is the main molecular mechanism of carbapenem resistance in K. pneumoniae in Asia, especially in China, and carriage of the $b l a_{\mathrm{KPC}-2}$ is mainly associated with strains of the clonal complex 258 (CC258). Among CC258, ST11 is the major sequence type (ST) from Asia, especially China (16).

The most abundant sequence type within this complex, ST258, emerged during the early to mid-2000s as an important human pathogen, descends from an ST11 ancestor which acquired a $\sim 1,100 \mathrm{~kb}$ genomic region from an otherwise distantly related ST442-like K. pneumoniae(17-20). In China, the "ancestral" ST11 remains as a dominant clonal lineage of carbapenemresistant $K$. pneumoniae, and forms the most common sequence type within CC258 in China (6, 13, 21-25).

Recently, a lineage of $K$. pneumoniae that simultaneously exhibits hypervirulence and carbapenem-resistance often refer to as "CR-hvKp" $(12,24,26-28)$. These strains are of major concern: not only are they hypervirulent and multidrug-resistant, but they are also highly transmissible $(24,25,29,30)$. Sequence analysis of a recent fatal outbreak of ST11 CR-hvKP strains in a Chinese hospital revealed that the several isolates belonged to a single clone, and yet with slight variation in pulsed-field gel electrophoresis (PFGE) patterns between the strains suggestive of extraordinarily high mutation rates (24). The observations in that study underscore the urgent need for a better understanding of CR-hvKp.

In this study, we describe an outbreak of ST11 K. pneumoniae strains that are both carbapenem-resistant and hypervirulent, isolated from the ICU of the First Affiliated Hospital of
Wenzhou Medical University. We sought to investigate the molecular and epidemiological features of these strains during the outbreak, especially on the characteristics of virulence factors and antimicrobial resistant determinants. The data from this outbreak confirmed that long-term carriage of CR-hvKp can give rise to in host evolution and an increase the repertoire of virulence factors carried. Hence, monitoring the nosocomial dissemination of CR-hvKp, providing effective strategies for antiinfective prophylaxis and outbreak management are essential.

\section{MATERIALS AND METHODS}

\section{Collection of $K$. pneumoniae Clinical Isolates From ICU}

The First Affiliated Hospital of Wenzhou Medical University is a leading teaching, three-grade hospital in Wenzhou, a city in Zhejiang province, China. The hospital features 3,000 beds, 64 departments and 89 wards. It is one of the largest health care centers in south Zhejiang province, has responsibility for the medical care of an estimated 30 million population.

A total of $29 \mathrm{~K}$. pneumoniae isolates were collected from eight patients from the ICU from March 2017 to January 2018. Patient information including age, gender, length of ICU stays, diagnosis, and outcomes is obtained from the Electronic Medical Records. The study and consent procedure were approved by the Ethical Committee of the First Affiliated Hospital of Wenzhou Medical University.

All isolates were isolated from various clinical specimens and identified as K. pneumoniae by VITEK-2 automated microbiology analyzer (bioMérieux, Marcy l'Etoile, France).

\section{Antimicrobial Susceptibility Testing (AST)}

Minimal inhibitory concentrations (MICs) of antimicrobial agents (gentamicin, tobramycin, ampicillin, cefazolin, ceftazidime, ceftriaxone, imipenem, ertapenem, levofloxacin, and ciprofloxacin) were determined by the agar dilution method and interpreted according to the Clinical and Laboratory Standards Institute (document M100-S27). Furthermore, ASTs of tigecycline and polymyxin B were performed by the broth microdilution method and interpreted by the recommendation of the European Committee on Antimicrobial Susceptibility Testing clinical breakpoints (http://www.eucast.org). Escherichia coli ATCC25922 and Pseudomonas aeruginosa ATCC27853 served as the quality control strains for susceptibility testing.

\section{Molecular Detection of Antibiotic Resistance Determinant}

Total DNA from each of the isolates was extracted from fresh bacterial colonies by using the Biospin Bacteria Genomic DNA Extraction kit (Bioflux, Tokyo, Japan). The presence of resistance determinants, including $\beta$-lactamase genes $\left(b l a_{\mathrm{CTX}-\mathrm{M}-1}\right.$ group, bla $a_{\mathrm{CTX}-\mathrm{M}-2}$ group, bla $a_{\mathrm{CTX}-\mathrm{M}-8}$ group, bla $a_{\mathrm{CTX}-\mathrm{M}-9}$ group, bla $a_{\mathrm{CTX}-\mathrm{M}-25}$ group, bla $a_{\mathrm{SHV}}, b l a_{\mathrm{TEM}}, b l a_{\mathrm{VEB}}, b l a_{\mathrm{CMY}}$, and $\left.b l a_{\mathrm{DHA}}\right)$, carbapenemase genes (bla $a_{\mathrm{KPC}}, b l a_{\mathrm{OXA}-23}, b l a_{\mathrm{SPM}}$, $b l a_{\mathrm{VIM}}, b l a_{\mathrm{GES}}, b l a_{\mathrm{NDM}-1}$, and $\left.b l a_{\mathrm{OXA}-48}\right)$, plasmid-mediated quinolone resistance (PMQR) genes ( $q n r A, q n r B, q n r C, q n r D$, $q n r S$, qepA, and $o q x A$ ), mutations in the GyrA and ParC proteins, 
the aminoglycoside resistance determinants $\left[\operatorname{aac}\left(3^{\prime}\right)-I a, \operatorname{aac}\left(6^{\prime}\right)\right.$ $I b$, and $\left.A N T\left(3^{\prime}\right)-I a\right], 16 S-R M T a s e$ genes ( $\left.r m t B, r m t C, \operatorname{armA}\right)$, and the polymyxin resistance gene $m c r-1$ were investigated by polymerase chain reaction (PCR) followed by sequencing. Primers used for PCR are shown in Table S2. The positive PCR products were sequenced by Beijing Genomics Institute Technology Co. Ltd. (Shanghai, China). Sequences alignments were completed by running BLAST at NCBI website (http://blast. ncbi.nlm.nih.gov/Blast.cgi).

\section{Detection of Virulence-Associated Features and Capsular Polysaccharide Synthesis (CPS) Genotyping}

The string test was used for screening for the hypermucoviscous phenotype as previously reported (31). Those strains with viscous string over $5 \mathrm{~mm}$ were considered as hvKP (2). PCR amplification was used to identify the capsular serotype of 29 isolates, including K-serotype-specific alleles K1, K2, K5, K20, K54, and K57, which were the most frequent genotypes in hvKp (32). Presence of 19 virulence-associated genes, including iut $A, f e p A, f y u A$, iroN, iroD, $\operatorname{rmp} A, \operatorname{rmp} A 2, \operatorname{mag} A, k f u B C, w c a G$, alls, ybtA, areA, uge, wabG, ent $B, f i m H, m r k D$, and $i u c A$ were detected in 29 isolates. To detect the existence of pLVPK-like plasmid, specific primers of repA, sopB, $L v 049$, Lv049 were designed based on the whole sequence previously reported (accession no. NC_005249.1). The specific primer sequences used in this study are listed in Table S1. The positive PCR products were sequenced by at Beijing Genomics Institute Technology Co. Ltd. (Shanghai, China). Nucleotide sequences alignments were compared completed by running BLAST at NCBI website (http://blast.ncbi.nlm.nih.gov/Blast.cgi).

\section{Molecular Epidemiology Analysis}

Multilocus sequence typing (MLST) was performed for phylogenetic analyses according to the protocols on the Pasteur Institute MLST website for K. pneumoniae (https://bigsdb. pasteur.fr/klebsiella/klebsiella.html).

All strains were subjected to PFGE analysis. Strain DNA were digested through $\mathrm{XbaI}$ for $2 \mathrm{~h}$ at $37^{\circ} \mathrm{C}$. Electrophoresis was conducted at $14^{\circ} \mathrm{C}$ for $18.5 \mathrm{~h}$ using the Bio-Rad ${ }^{\circledR}$ CHEF-Mapper XA machine (Bio-Rad, California, USA). Salmonella enterica serotype Braenderup H9812 was used as the molecular marker (33). Reports-phylogenetic tree (UPGAMA) was constructed to determine the hierarchical representation of different linkage levels between the strains. Chromosomal DNA restriction patterns were interpreted using previously established guidelines (34), with a phylogenetic dendrogram analysis indicated at $85 \%$ demonstrating strain relatedness.

\section{Galleria mellonella Larvae Infection Assay}

The virulence capability was evaluated through the commonly used in vivo infection model, the wax moth Galleria mellonella(7, 24, 35-37). According to the antimicrobial resistant profile and hypermucoviscous genotype reported previously, five strains were picked out for the further infection model. Two strains (Kp1 and Kp15) were resistant and hypermucoviscous as well, two strains (Kp4 and Kp21) were resistant but nonhypermucoviscous and one (Kp6) was susceptible and nonhypermucoviscous. The methods were described previously (35). In the present study, each infection group includes eight larvae and were injected with $10 \mu \mathrm{L}$ of bacterial suspension with bacteria concentration ranging from $10^{5}$ to $10^{7} \mathrm{CFU}$ per larva. As a control group, $10 \mu \mathrm{L}$ PBS was injected in parallel. All larvae were placed in Petri-dishes and kept at $37^{\circ} \mathrm{C}$ in the dark. The number of dead larvae was recorded with notes on any melanization and lack of motility 24, 48, and $72 \mathrm{~h}$ after injection, respectively. All experiments were repeated in biological triplicate as described and results were not considered if two or more larvae in any of the control groups died. The survival curves were plotted by the GraphPad Prism 5.0 program and the Kaplan-Meier method was used for statistical analysis. $P$ $<0.05$ was considered statistically significant. For each isolate, the $50 \%$ lethal dose $\left(\mathrm{LD}_{50}\right)$ was analyzed using the probit analysis program by SPSS 22.0 and the results were expressed as $\log _{10} \mathrm{LD}_{50}$.

\section{RESULTS}

\section{Outbreak Description}

From March 2017 to January 2018, eight patients (age range 13-69 years, ratio male-female 3:1) had fatal nosocomial infections during the period of hospitalization in the ICU. The eight patients died of respiratory failure, septic shock, and multiple organ failure (Table 1). All patients had submitted to invasive procedures (including surgery, endotracheal intubation, thoracentesis, urinary catheterization, laparoscopy, and gastric intubation) before the first $K$. pneumoniae was isolated in ICU (Figure 1). Patients diagnoses varied as pulmonary infection $(4 / 8,50 \%)$, post-operative infection $(2 / 8,25 \%)$, hemorrhagic infarct $(1 / 8,12.5 \%)$, and polytrauma $(1 / 8,12.5 \%)$. This event was characterized as an outbreak and attracted the attention of the hospital infection-control department.

The retrospective clinical data showed that the first case (patient 8) was a male patient admitted to the ICU on 25th March 2017 with the diagnosis of pulmonary infection. On day first after hospitalization, three ST11 K. pneumoniae (Kp27, $\mathrm{Kp} 28$, and Kp29) isolates were retrieved from fecal, pus and sputum samples, respectively, and the patient died of fatal infection at day 15 (Figure 1, Table 1). The second case was patient 4, a 69-year-old man who underwent surgery for a brainstem tumor, and was admitted to the ICU on 1st June 2017 (Figure 1, Table 1). Patient 4 had the longest hospitalization length of stay (228 days), dating from June 2017 to January 2018. On 2nd June, K. pneumoniae was isolated from a sputum sample from patient 4 . In the course of the ICU stay, patient 4 yielded 11 independent Klebsiella samples, with positive sputum samples recovered for 115 days (Figure 1). Administration of antibiotics (cefoperazone, meropenem, and tigecycline) did not resolve the ongoing respiratory infection. Two fecal samples from patient 4, in June and November, were also positive for Klebsiella (Figure 1) but were subsequently identified as a distinct sequence type (ST37, Figure 2), while other isolates belonged to ST11. Ultimately, an ST11 strain was isolated from 
TABLE 1 | Characteristics of the eight patients and the isolated $K$. pneumoniae strains.

\begin{tabular}{|c|c|c|c|c|c|c|c|c|c|}
\hline Patient & Age & $\begin{array}{l}\text { Length of } \\
\text { stay (Days) }\end{array}$ & Diagnosis & Outcc & Isolate & Isolate date & $\begin{array}{l}\text { Isolate } \\
\text { type }\end{array}$ & $\begin{array}{c}\text { ST } \\
\text { type }\end{array}$ & $\begin{array}{l}\text { PFGE } \\
\text { cluster }\end{array}$ \\
\hline \multirow[t]{2}{*}{ P1 } & $50-60$ & 28 & Post-operation of cholelithiasis & Died & Kp1 & 31 October & Blood & 11 & $E$ \\
\hline & & & & & Kp2 & 3 November & Sputum & 11 & $E$ \\
\hline \multirow[t]{3}{*}{ P2 } & $50-60$ & 31 & Pulmonary infection & Died & Kp3 & 12 November & Fecal & 11 & $E$ \\
\hline & & & Brain infarct & & Kp4 & 29 November & Urine & 11 & $E$ \\
\hline & & & & & Kp5 & 5 December & Fecal & 11 & $E$ \\
\hline \multirow[t]{2}{*}{ P3 } & $50-60$ & 17 & Polytrauma & Died & Kp6 & 11 December & Sputum & 375 & $\mathrm{~B}$ \\
\hline & & & & & Kp7 & 20 December & Blood & 11 & C \\
\hline \multirow[t]{11}{*}{ P4 } & $60-70$ & 228 & Pulmonary infection & Died & Kp8 & 2 June & Sputum & 11 & $E$ \\
\hline & & & Brain stem tumor operation & & Kp9 & 22 June & $\begin{array}{l}\text { Wound } \\
\text { exudate }\end{array}$ & 11 & $E$ \\
\hline & & & & & Kp10 & 23 June & Fecal & 37 & A \\
\hline & & & & & Kp11 & 30 June & Urine & 11 & $E$ \\
\hline & & & & & Kp12 & 30 June & Sputum & 11 & $E$ \\
\hline & & & & & Kp13 & 6 July & Sputum & 11 & $E$ \\
\hline & & & & & Kp14 & 11 August & Sputum & 11 & $E$ \\
\hline & & & & & Kp15 & 24 September & Sputum & 11 & $E$ \\
\hline & & & & & Kp16 & 17 November & Fecal & 37 & $A$ \\
\hline & & & & & Kp17 & 1 January & Fecal & 11 & $E$ \\
\hline & & & & & Kp18 & 13 January & Blood & 11 & $E$ \\
\hline \multirow[t]{3}{*}{ P5 } & $60-70$ & 34 & Obstructive jaundice & Died & Kp19 & 13 December & Blood & 11 & $\mathrm{D}$ \\
\hline & & & Tumor of duodenum & & Kp20 & 15 December & $\begin{array}{l}\text { Drainage } \\
\text { fluid }\end{array}$ & 11 & $\mathrm{D}$ \\
\hline & & & & & Kp21 & 19 December & Catheter & 11 & $\mathrm{D}$ \\
\hline \multirow[t]{2}{*}{ P6 } & $10-20$ & 10 & Pulmonary infection & Died & Kp22 & 15 November & Sputum & 11 & $E$ \\
\hline & & & & & Kp23 & 17 November & Urine & 11 & $E$ \\
\hline \multirow[t]{3}{*}{ P7 } & $60-70$ & 80 & Hemorrhagic infarct & Died & Kp24 & 20 January & Fecal & 11 & $\mathrm{D}$ \\
\hline & & & & & Kp25 & 9 February & Sputum & 11 & $\mathrm{D}$ \\
\hline & & & & & Kp26 & 13 February & Urine & 11 & $\mathrm{D}$ \\
\hline \multirow[t]{3}{*}{ P8 } & $60-70$ & 15 & Pulmonary infection & Died & Kp27 & 26 March & Fecal & 11 & $E$ \\
\hline & & & & & Kp28 & 26 March & Pus & 11 & $E$ \\
\hline & & & & & Kp29 & 26 March & Sputum & 11 & $E$ \\
\hline
\end{tabular}

the patient's blood in January 2018, immediately preceding death. Within this time frame, ST11 K. pneumoniae were isolated from other six patients in the ICU concurrently during this hospitalization period (Figure 1), indicating a potential of nosocomial transmission. The infection timeline is detailed in Figure 1.

To control this outbreak, all patients were placed in contact isolation. The patients were arranged in single rooms with disinfection of $2 \%$ chlorhexidine baths daily and the affected patients' rooms were disinfected by glutaraldehyde thoroughly. The staff members in the ICU were screened for signs and symptoms of infections daily. The outbreak was effectively controlled and besides these eight patients, no further infected patients were found in the ICU.

\section{Epidemiological Analysis and Virulence Characteristics}

Multilocus sequence typing (MLST) analysis revealed that 26 isolates $(26 / 29,89.7 \%)$ retrieved from eight patients were identified as ST11 K. pneumoniae, indicating that this was the dominant ST type in these ICU deaths (Figure 2). In addition, three isolates belonged to either ST37 (patient 4, Kp10, Kp16; $6.9 \%$ ) or ST375 (patient 3, Kp6; 3.4\%). The similarities seen in the MLST phylogram indicated close relationships between some of the isolates. For example, the Kp1 isolate from the initial blood sample drawn from patient 1 was very closely related to the isolates $\mathrm{Kp} 2$ and $\mathrm{Kp} 3$ (Figure 2) drawn from both patient 1 and patient 2 in November (Figure 1). The subsequent urinary tract infection of patient 2 in December (Figure 1) was attributable to a distinct pair of ST11 isolates, Kp4, and Kp5 (Figure 2). The PFGE results showed that 29 isolates were divided into five different PFGE clusters (A to E). Cluster D and E accounted for 20.6\% (6/27) and $65.5 \%(19 / 27)$ of ST11 isolates, respectively, indicating that these strains were highly homologous. The two ST37 isolates belonged to the same cluster (cluster A). Kp6 (ST375) and Kp7 (ST11) formed separate monotypic clusters, cluster B and C, respectively (Figure 2). 


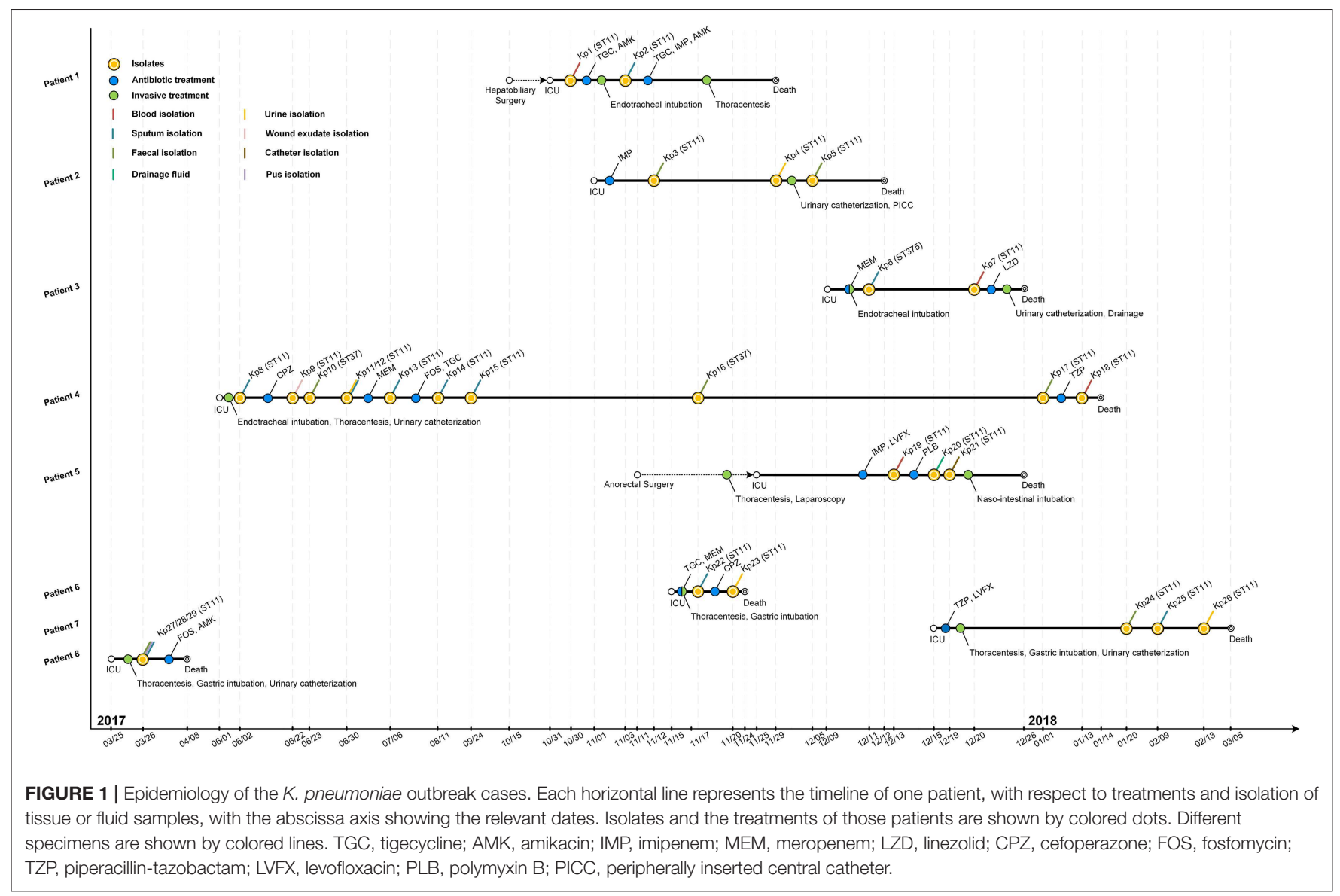

According to previous definition, the ST11 K. pneumoniae should be considered potentially hypervirulent $(23,24,38)$. This, together with the high mortality rate ( $8 / 8$ patients, $100 \%)$ in the outbreak led us to address the characteristics of these new isolates.

\section{Extracellular Capsule}

Only two isolates $(\mathrm{Kp} 2, \mathrm{Kp} 7)$ lacked the transcriptional regulator of mucoid phenotype rmpA, a gene found responsible for upregulation of the synthesis of capsular polysaccharide (39). All of the isolates were negative for $r p m A 2$, which has been reported as a trans-acting activator for the capsular polysaccharide biosynthesis (39). The wzy-like gene magA was involved in polysaccharide synthesis in K1 capsulated strains (40), and the biofilm-promoting fucose synthase $w c a G$ (41) is required for decoration of the capsule polysaccharide with fucose. Both genes were absent from all isolates (Figure 3). While the ST11 strains were of indeterminate capsule type (i.e., not of the typical K1 capsule type) and only Kp6 (ST375) was identified as K2 capsule type (Figure 3). Interestingly, only four ST11 (Kp1, Kp6, Kp15, and Kp18) were string-test positive, which was correlated with a hypermucoviscous phenotype in previous studies $(2,43)$.

\section{Iron Acquisition}

All ST11 isolates except Kp8, Kp11, and Kp21 were positive for $i u t A$, most isolates $(23 / 29,79.3 \%)$ were positive for $i u c A$, indicating the existence of aerobactin uptake, the dominant component of the siderophore system involved in hypervirulence (44). All isolates were positive for entB and 25 isolates were positive for fepA, consistent with the use of the siderophore enterobactin for iron acquisition, and most ST11 isolates were also positive for $y b t A(25 / 26)$ and fyuA (23/26), demonstrating that they could also use yersiniabactin for iron acquisition (Figure 3). Only some of the ST11 isolates had acquired the genes $k f u B C$ encoding a ferric iron uptake $\mathrm{ABC}$ transporter. The ST375 isolate (Kp6) harbored siderophore uptake or biosynthesis genes: aerobactin (iutA), salmochelin (iroD), enterobactin (fepA), and yersiniabactin $(f y u A)$, which are commonly associated with hypervirulence [Figure 3; (44)].

\section{Fimbrial Adhesin}

All ST11 isolates were positive for fim $H$ and $m r k D$ suggesting that they can express type 1 and type 3 fimbrial adhesion $(45,46)$, which mediate binding to the extracellular matrix and play an important role in biofilm formation (47).

\section{Nitrogen Metabolism}

All isolates were found to be negative for the alls transcriptional activator (Figure 3), suggesting they were incapable of allantoin utilization to compete for nitrogen access in host niches (48). Most of the isolates were positive for ureA (23/29), which enables the use of urea as a nitrogen source in host niches (49). 


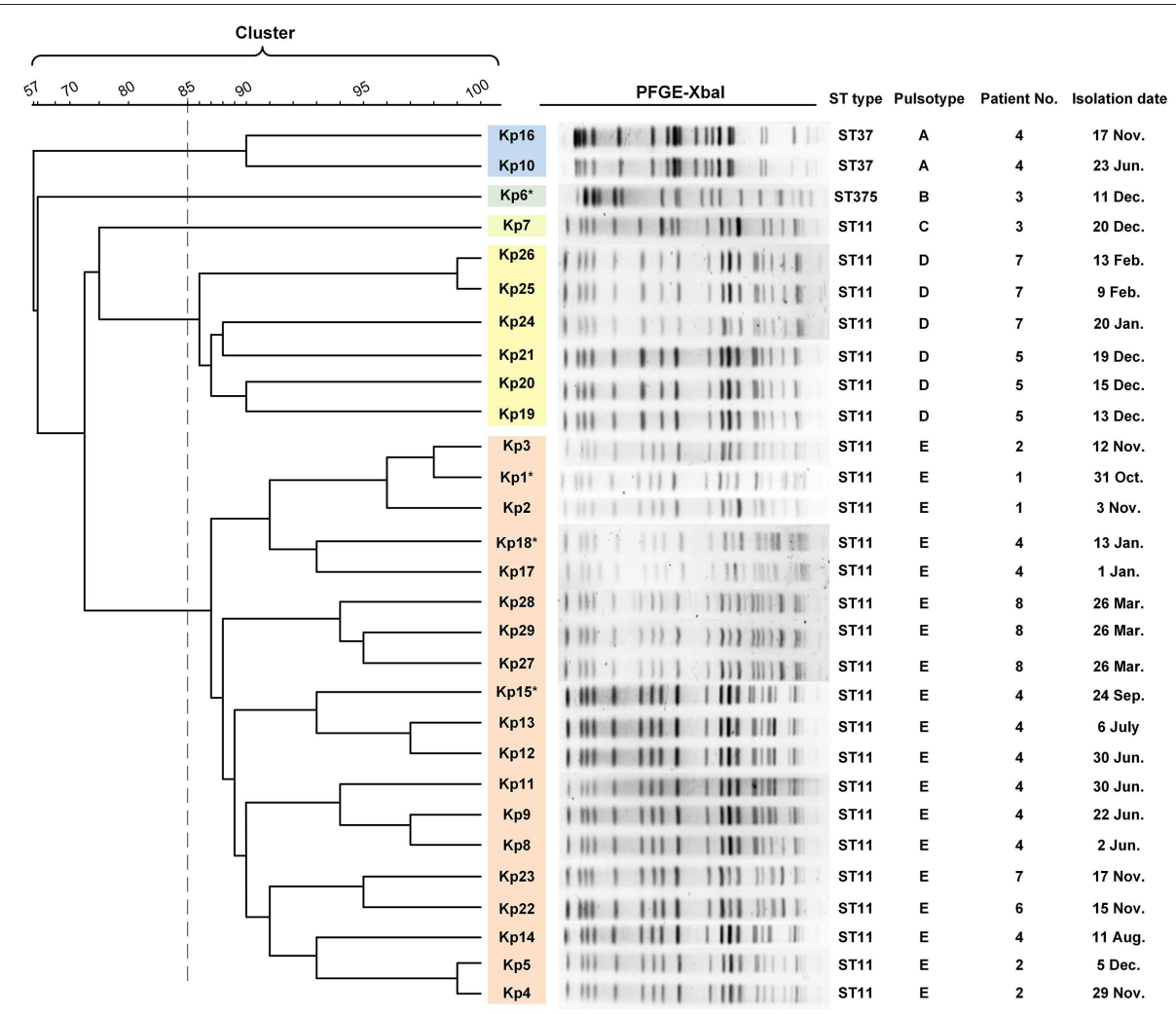

FIGURE 2 | PFGE results and MLST typing for 29 K. pneumoniae isolates. Genomic DNA from each of the 29 K. pneumoniae isolates was digested using Xbal and the digests subjected to pulsed-field gel electrophoresis to generate diagnostic genomic DNA fragmentation fingerprints. The dendrogram of the PFGE profiles is clustered by the UPGAMA on the basis of the Dice similarity by the Quantity One software package 4.6. The sequences of seven housekeeping genes (gapA, infB, $m d h, p g i, p h o E, r p o B$, and tonB) were analyzed for the purpose of MLST typing. PFGE results showed that 29 isolates were divided into five different PFGE clusters (A-E) as represented by different colors. Cluster D and E accounted for 20.6\% (6/27) and 65.5\% (19/27) of ST11 isolates, respectively, indicating that those strains were highly homologous. The two ST37 isolates belonged to the same cluster (cluster A). Kp6 (ST375) and Kp7 (ST11) formed separate monotypic clusters, cluster B and $\mathrm{C}$, respectively. Asterisk indicates the string test of isolate was positive.

Besides, The pLVPK-like plasmid carrying capsular polysaccharides regulator genes $(r m p A$ and $r m p A 2)$ and several siderophore gene clusters is known to contribute to the virulence of hvKp (24). Our results describe that while most isolates were positive for iucA (23/29, 79.3\%), repA $(28 / 29$, $96.6 \%), \operatorname{sopB}(28 / 29,96.6 \%), L v 049$ (24/29, 82.8\%), and Lv204 $(26 / 29,89.7 \%)$, they were negative for $r m p A 2$. Thus, consistent with a very recent study of ST23 K. pneumoniae in Japan (50), we inferred that the outbreak isolates might carry a modified version of the pLVPK plasmid.

\section{Carbapenem-Resistance and $\beta$-Lactamase Genes}

The clinical break-point for carbapenem in the treatment of Klebsiella is $2 \mu \mathrm{g} / \mathrm{mL}$ (ertapenem) or $4 \mu \mathrm{g} / \mathrm{mL}$ (doripenem, imipenem and meropenem) (CLSI-M100, 2017). Isolates from patients 1 to 6, who did not respond to treatment with carbapenems, were confirmed ex vivo to be resistant to the carbapenems imipenem and ertapenem (MIC ranged from 8 to $>128 \mu \mathrm{g} / \mathrm{mL}$ ). With the exception of Kp6 (ST375), which did not display a multidrug-resistant phenotype, all other isolates carried the $b l a_{\mathrm{KPC}-2}$ gene (Figure 3, Table S1). All isolates were negative for $b l a_{\mathrm{NDM}-1}$ and $b l a_{\mathrm{OXA}-48}$. Excluding Kp6, all isolates were resistant to $\beta$-lactams, including ampicillin, cefazolin, ceftazidime, and ceftriaxone (MIC > $>4 \mu \mathrm{g} / \mathrm{mL}$ ). The other $\beta$-lactamase genes detected, including bla $a_{\mathrm{SHV}-11}$ (28 isolates), bla $a_{\mathrm{CTX}-\mathrm{M}-2}$ group (27 isolates), bla $a_{\mathrm{CTX}-\mathrm{M}-9}$ group (23 isolates), $b l a_{\mathrm{TEM}-1}$ (10 isolates), bla $a_{\mathrm{CMY}-2}$ (seven isolates), and bla $a_{\mathrm{DHA}}$ (four isolates). These genes were distributed widely, but not uniformly, across the ST11 isolates (Figure 3, Table S1).

\section{Distribution of Other Drug-Resistance Mechanisms}

Of the $29 \mathrm{~K}$. pneumoniae isolates, 22 (75.9\%) exhibited high-level resistance to the aminoglycoside gentamicin $(32->128 \mu \mathrm{g} / \mathrm{mL})$, and $22(75.9 \%)$ showed high-level resistance toward the aminoglycoside tobramycin $(>128 \mu \mathrm{g} / \mathrm{mL})$. This is consistent with observations that 24 isolates carried various aminoglycoside resistance determinants, including $a a c\left(3^{\prime}\right)-I a$ and $a a c\left(6^{\prime}\right)-I b$ each of which encodes aminoglycoside-modifying enzyme, ANT(3')Ia (encoding an aminoglycoside adenyltransferase), $r m t B$, and $r m t C$ each of which encodes a distinct $16 \mathrm{~S}$ rRNA methylase 


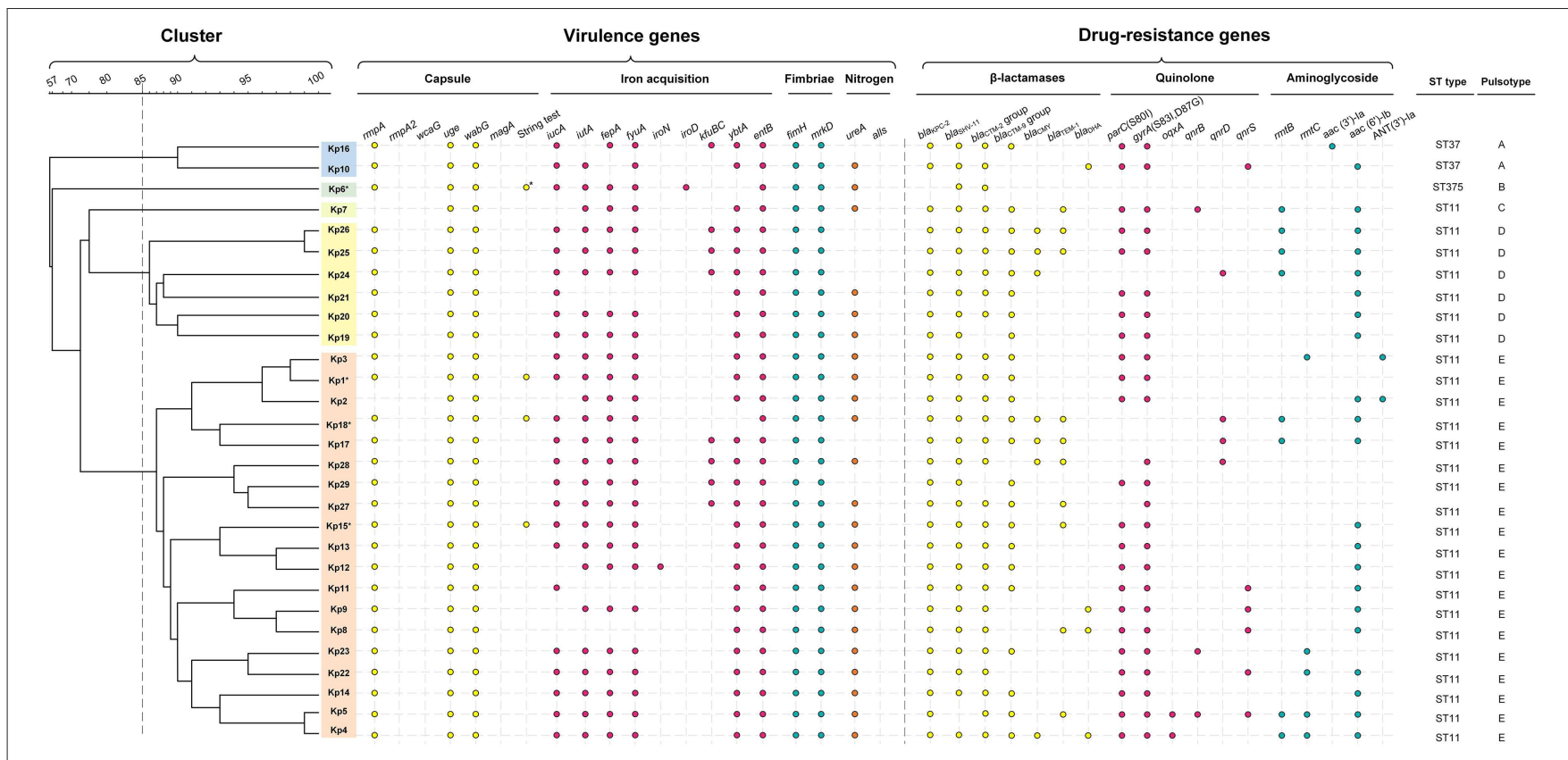

FIGURE 3 | Gene profiles of $K$. pneumoniae isolates. Virulence genes and drug-resistance genes identified by PCR-based profiling are shown by colored dots, plotted against the phylogeny of the strains from the whole genome PFGE analysis shown in Figure 2. Asterisk indicates the capsule serotype was determined to be of K2 type. The relevant abbreviations are: bla, beta-lactamases (specific isoforms bla $\mathrm{KPC}_{-2}$, bla $\mathrm{SHV}_{-11}$, bla $\mathrm{CTX}-\mathrm{M}-2$ group, bla $\mathrm{CTX}-\mathrm{M}-9$ group, bla $\mathrm{CMY}-2$, bla $\mathrm{TEM}-1$, bla $\mathrm{DHA}_{\mathrm{A}}$ ); mutations in gyrA (S83l, D87G) and parC (S80I), and the presence of qnrB, qnrS, and oqXA all reduce susceptibility to fluoroquinolones (42); rmtB, $r m+C$ (encoding 16S rRNA methylases), aac $\left(3^{\prime}\right)-l a$, aac $\left(6^{\prime}\right)-1 b$, and $A N T\left(3^{\prime}\right)-l a$ (encoding aminoglycoside-modifying enzymes) are all aminoglycoside resistance genes.

that modifies ribosomes and causes resistance to aminoglycosides (Figure 3).

Most $(27 / 29,93.1 \%)$ isolates were resistant to the quinolones levofloxacin and ciprofloxacin (MIC ranged from 16 to $128 \mu \mathrm{g} / \mathrm{mL}$ ), except for Kp16, which was judged intermediate in resistance to levofloxacin and ciprofloxacin (MIC $=4 \mu \mathrm{g} / \mathrm{L})$, and Kp6, which was susceptible to both of them (MIC $\leq 0.25 \mu \mathrm{g} / \mathrm{mL}$ ). Resistance to fluoroquinolones was largely due to point mutations; 25 isolates carried missense quinolone-resistance mutations in the DNA replication machinery, including parC (24 isolates: S80I in DNA topoisomerase IV) and gyrA (25 isolates: S83I, D87G in DNA gyrase). In addition, plasmid-mediated quinolone resistance determinants $q n r B, q n r D$, qnrS, and $o q x A$ were also determined in several isolates (Figure 3).

Polymyxin-resistance was measurable in two of the isolates, Kp12 (patient 4, ST11) and Kp21 (patient 5, ST11), but not in any others. Nevertheless, these two strains were negative for $m c r-1$, the most prominent cause of polymyxin resistance in China $(51,52)$. However, other resistance mechanism such as modifications of two-component systems (PmrA/PmrB and PhoP/PhoQ) and inactivation of the $\operatorname{mgrB}$ gene (53) might be present, but would require to be further investigated. Interestingly, patient 5 did not respond to polymyxin treatment, despite Kp19 (ST11) being deemed polymyxin-sensitive ex vivo (Table S3). Similarly, for the glycylcycline antibiotic tigecycline, patients 1,4 , and 6 did not respond to intravenous treatment with the antibiotics, despite all isolates being deemed sensitive to tigecycline ex vivo (Table S3).

\section{Hypervirulence Measurements Through the Death of G. mellonella Larvae}

Five isolates were collected based on the antimicrobial resistance profile and hypermucoviscous phenotype: Kp6 (patient 3, ST375, hypermucoviscous, antibiotic-sensitive), Kp1 (patient 1, ST11, hypermucoviscous, carbapenem-resistant, bla $a_{\mathrm{KPC}-2}$ positive), and Kp15 (patient 4, ST11, hypermucoviscous, carbapenemresistant, bla $a_{\mathrm{KPC}-2}$ positive), Kp4 (patient 2, ST11, nonhypermucoviscous, carbapenem-resistant, bla $a_{\mathrm{KPC}-2}$ positive), and Kp21 (patient 5, ST11, non-hypermucoviscous, carbapenemresistant, $b l a_{\mathrm{KPC}-2}$ positive). Among the five selected strains, the $\mathrm{Kp} 1$ isolate from the initial blood sample drawn from patient 1 is hypermucoviscous as determined by string-test and resistant to beta-lactams, carbapenem and quinolones. During the outbreak this strain was most likely responsible for the infection of patient 1 possibly transmitted to patient 2 (Figure 1, Table S3). The isolate Kp6 was ST375, showed a positive string test as well as acquisition of genes for iutA, which predicted it could be hypervirulent. Kp21 was isolated from a urinary catheter of patient 5 before expiration. From our analysis it was determined to be non-hypermucoviscous, but extremely drug-resistant. It was therefore of interest to determine the virulence phenotypes of Kp1, Kp6, and Kp21. In addition, Kp4 and Kp15 were selected randomly from each of the two groups with hypermucoviscous phenotype and non-hypermucoviscous phenotype.

All isolates caused a time-dependent death of the larvae. When the dose of bacteria was altered $\left(10^{5}, 10^{6}, 10^{7} \mathrm{CFU}\right)$, K. pneumoniae-induced lethality was found to be dependent 
on the inoculum density (Figure S1). After $72 \mathrm{~h}$ of infection, the mortality of the larvae infected with $10^{7} \mathrm{CFU}$ bacterial suspension was higher than that infected with $10^{5} \mathrm{CFU}$ bacterial suspension. The observed mortality of the larvae was higher than $60 \%$ after $72 \mathrm{~h}$ (Figure 4 ) with the most virulent being Kp4 (100\% mortality), Kp21 (100\% mortality), and Kp6 (75\% mortality). There was no significant difference among these groups $(P>0.05)$.

The recent assessment of a range of $K$. pneumoniae isolates suggests the parameters for the Galleria model to define hypervirulence, based on a calculation of $\mathrm{LD}_{50}$ value (37). In Shi's study, isolates of $K$. pneumoniae generating an $\mathrm{LD}_{50}<5.06 \log _{10}$ $\mathrm{CFU} / \mathrm{ml}$ were defined as hypervirulent. According to that study, we calculated $\mathrm{LD}_{50}$ for $\mathrm{Kp} 1\left(5.324 \log _{10} \mathrm{CFU} / \mathrm{ml}\right), \mathrm{Kp} 4$ (5.755 $\left.\log _{10} \mathrm{CFU} / \mathrm{ml}\right), \mathrm{Kp6}\left(4.954 \log _{10} \mathrm{CFU} / \mathrm{ml}\right), \mathrm{Kp} 15$ (5.017 $\log _{10}$ $\mathrm{CFU} / \mathrm{ml})$, and Kp21 (4.266 $\left.\log _{10} \mathrm{CFU} / \mathrm{ml}\right)$.

\section{DISCUSSION}

Our study reported an outbreak of ST11 K. pneumoniae in the ICU of a teaching hospital where multiple isolates showed highly multiple-drug resistance phenotypes including carbapenem-resistance. Sampling from patients showed continuing colonization with K. pneumoniae. Isolates were taken under a surveillance protocol aimed at patient care, which means sampling bias interferes with drawing strong conclusions on the extent of in host evolution vs. transmission and re-infection by multiple ST11 K. pneumoniae strains. Nonetheless, several important points can be made from the data gathered from this ICU outbreak.

Firstly, this study is consistent with observations of long-term colonization by carbapenem-resistant $K$. pneumoniae reported by three other studies of hospital-acquired infection (54-56). As the ICU outbreak reported in the United States of America, two patients who survived the outbreak remained colonized by

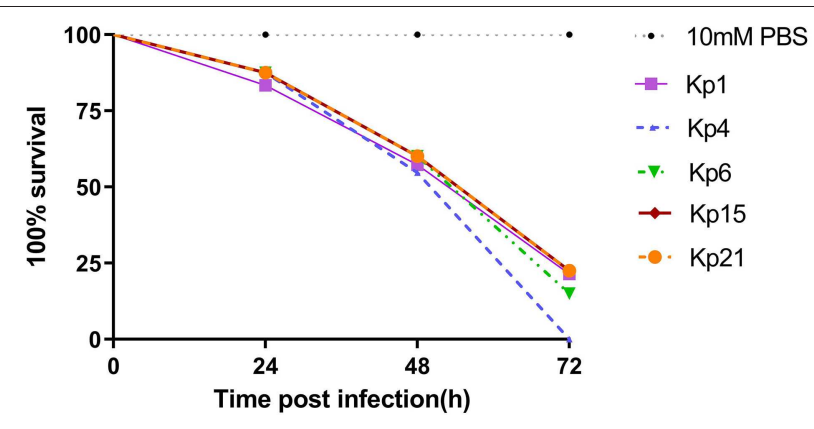

FIGURE 4 | K. pneumoniae infection of G. mellonella larvae induces time-dependent lethality. The effect of $1 \times 10^{6}$ colony-forming units (CFU) of each K pneumoniae strain on survival was observed in G. mellonella. The strains were divided into three groups according the hypermucoviscous and resistance phenotypes. High-level drug resistant and hypermucoviscous (Kp1, Kp15). High-level drug resistant and non-hypermucoviscous (Kp4, Kp21). Drug sensitive but hypermucoviscous (Kp6). Larvae were injected with PBS or with $10^{6} \mathrm{CFU}$ of $\mathrm{Kp} 1, \mathrm{Kp} 4, \mathrm{Kp} 6, \mathrm{Kp} 15$, and Kp21. The survival was monitored over $72 \mathrm{~h}$ post-infection. Mortality of larvae infected with Kp1, Kp4, Kp6, Kp15, and Kp21 were time-dependent. carbapenem-resistant K. pneumoniae for up to 4 years. A single clone was detected in samples from various sites (throat, groin, rectum) of the patients, and the documented characteristics of in host evolution over these years suggested recombination and gene loss events, particularly affecting the plasmids carried by the long-term colonizing K. pneumonia (56). In this outbreak in the First Affiliated Hospital in Wenzhou, patient 4 remained colonized by carbapenem-resistant K. pneumoniae, with positive sputum samples recovered for 115 days.

Secondly, most K. pneumoniae $(26 / 29,89.7 \%)$ in this outbreak were assigned to ST11 lineage which was reported as being prevalent in the wide spread of KPC-producing K. pneumoniae in China (13). In early 2011, a nosocomial outbreak of bla $a_{\mathrm{KPC}-2}$ positive ST11 CRKP infection was observed in the north of China (57). Subsequently, several outbreaks of bla $a_{\mathrm{KPC}-2}$ positive ST11 CRKP were reported in multiple hospitals in China (5861 ), echoing the prevalence of those stains in the mainland of China. In our study, all ST11 CRKP harbored bla $a_{\mathrm{KPC}-2}$ and bla $a_{\mathrm{SHV}-11}$, and most were also positive for bla $a_{\mathrm{TEM}-1}$ and bla $a_{\mathrm{CTX}-\mathrm{M}}$, consistent with previous reports. ST11 CR-hvKP has been recently reported elsewhere in China, causing fatal infections and high mortality in other hospitals $(24,38,61$, 62).This seemingly recent increase in occurrence of the ST11 CR-hvKP in various Chinese hospitals is of grave concern, as it indicates either the presence of these strains within the community, or the high potential of ST11 K. pneumoniae to repeatedly yet independently acquire hypervirulence genes in the distinct hospitals to cause serious outbreaks. The isolates presented in our study carried more resistance determinates in their genotypes, and showed correspondingly more multi-drug resistant phenotypes, compared to other reports in the literature. This indicates that increasingly serious threats are emerging.

Thirdly, our study highlights some short-comings in realtime diagnostics of drug-resistant phenotypes and genotypes in the case of $K$. pneumoniae detection. Technologically, it is now feasible to apply partial-targeted or whole genome sequencing (WGS) to patient samples in order to determine drug resistance profiles. However, there were multiple instances in this outbreak where isolated bacteria were sensitive to the antibiotic used ex vivo, nevertheless, the infection caused by these isolates did not respond to antibiotic treatments effectively in vivo. For example, in the case of patient 4 administration of antibiotics including tigecycline was ineffective in treating the respiratory infection, despite the observation that ex vivo, the isolates from patient 4 were tigecycline-sensitive. Another example was in the case of patient 5 , polymyxin $B$ also failed to treat the infection although the isolates was sensitive ex vivo. For future outbreaks, introducing WGS analysis should be considered as it can identify pathogenic virulence factors, diagnostic for hvKP variants guiding specific hospital outbreak control measures and treatment plans to be actioned (24, 60, $62,63)$. For instance, due to the high transmission nature of hvKP $(24,64)$, time-sensitive treatment and strict isolation measures should be performed immediately after detection to prevent further possible transmission reducing. Furthermore, as hvKP often present as abscesses, drainage of these sites of infection should be considered (2), and strict glycemic control 
may help clinicians to reduce the possibility of septic metastatic complications (43).

Finally, with the emergence of drug-resistances in hypervirulent strains of $K$. pneumoniae, it is incumbent on the community to have clearer definitions of the term "hypervirulence". The accepted clinical definition of hvKP was based on manifestations that include liver abscesses, metastatic meningitis and endophthalmitis (2). In some studies, hypervirulent strains were identified by their hypermucoviscous phenotype through string test $(26,38,65)$. Alternatively, hypervirulence gene signatures have been developed through genome-wide association studies that mostly identified an increased capability of iron acquisition and likely production of a hypermucoviscous phenotype $(2,6-8,11,12,66)$. Ultimately, the definition of a hypervirulent phenotype is a greatly increased level of virulence. Larvae from Galleria mellonella, a wax moth, are becoming more widely used to study the pathogenesis of $K$. pneumoniae infections and the efficacy of antimicrobial drugs and phage-based therapies $(24,35-37,67,68)$. It is an experimentally tractable model and the similarities between these insects and vertebrate innate immune responses make it suitable for screening and comparative analysis of bacteria isolated from human patients (69). The results presented here show that the $K$. pneumoniae infection of G. mellonella larvae induced dose-dependent and time-dependent lethality. Importantly, even with strains that covered a range of drugresistance and hypermucoviscous phenotypes, similarly high mortality rates were observed in the animal model of infection, with no significant difference between these isolates. This data suggests that hypervirulence is not directly predictable from genetic signatures indicative of hypermucoviscosity or iron-acquisition phenotypes. Our data support the proposition that "hypervirulent" could be defined through calculated $\mathrm{LD}_{50}$ value drawn from a standardized Galleria model assay (37). The threshold value used to denote hypervirulence should be addressed by the Klebsiella research community.

In conclusion, our study described the outbreak of ST11 K. pneumoniae that exhibited hypervirulence and multidrug

\section{REFERENCES}

1. Meatherall B, Gregson D, Ross T, Pitout J, Laupland K. Incidence, risk factors, and outcomes of Klebsiella pneumoniae bacteremia. Am J Med. (2009) 122:866-73. doi: 10.1016/j.amjmed.2009.03.034

2. Shon A, Bajwa R, Russo T. Hypervirulent (hypermucoviscous) Klebsiella pneumoniae: a new and dangerous breed. Virulence. (2013) 4:107-18. doi: $10.4161 /$ viru. 22718

3. Mathers A, Peirano G, Pitout J. The role of epidemic resistance plasmids and international high-risk clones in the spread of multidrugresistant Enterobacteriaceae. Clin Microbiol Rev. (2015) 28:565-91. doi: 10.1128/CMR.00116-14

4. Fung C, Chang F, Lee S, Hu B, Kuo B, Liu C, et al. A global emerging disease of Klebsiella pneumoniae liver abscess: is serotype $\mathrm{K} 1$ an important factor for complicated endophthalmitis? Gut. (2002) 50:420-4. doi: 10.1136/gut.50.3.420

5. Turton J, Perry C, Elgohari S, Hampton C. PCR characterization and typing of Klebsiella pneumoniae using capsular type-specific, variable number resistance in one of the largest comprehensive hospitals in China. With the prevalence of such CR-hvKp, severely challenges have appeared in clinical treatments and pose a substantial threat to the world's population. Sustained efforts are required to limit the transmission of these strains, and prevent future outbreaks.

\section{DATA AVAILABILITY}

The raw data supporting the conclusions of this manuscript will be made available by the authors, without undue reservation, to any qualified researcher.

\section{AUTHOR CONTRIBUTIONS}

$\mathrm{XZ}, \mathrm{YaZ}, \mathrm{WB}$, and YiZ contributed to the design of the experiments. XZ, YiZ, and YaZ performed the experiment. TL and YaZ wrote the initial draft of the manuscript. XZ, YiZ, LC, and YaZ contributed to the acquisition, analysis, and interpretation of the data included in this manuscript. VT, AR, JL, RS, SZ, TZ, and JC revised the manuscript. JW revised the figures. All authors approve of the final manuscript being submitted and agree to be accountable for the work detailed in the submitted manuscript.

\section{FUNDING}

This work was supported by research grants from the National Natural Science Foundation of China (No. 81741059); Program Grant 1092262 from the National Health and Medical Research Council of Australia (NHMRC); and by the Zhejiang Provincial Program for the Cultivation of High-level Innovative Health Talents (No. [2012]241).

\section{SUPPLEMENTARY MATERIAL}

The Supplementary Material for this article can be found online at: https://www.frontiersin.org/articles/10.3389/fpubh. 2019.00229/full\#supplementary-material tandem repeat and virulence gene targets. J Med Microbiol. (2010) 59:541-7. doi: 10.1099/jmm.0.015198-0

6. Struve C, Roe C, Stegger M, Stahlhut S, Hansen D, Engelthaler D, et al. Mapping the evolution of hypervirulent Klebsiella pneumoniae. MBio. (2015) 6:e00630. doi: 10.1128/mBio.00630-15

7. Lam MMC, Wyres KL, Duchêne S, Wick RR, Judd LM, Gan YH, et al. Population genomics of hypervirulent Klebsiella pneumoniae clonal-group 23 reveals early emergence and rapid global dissemination. Nat Commun. (2018) 9:2703. doi: 10.1038/s41467-018-05114-7

8. Lai Y, Lin A, Chiang M, Dai Y, Hsu C, Lu M, et al. Genotoxic Klebsiella pneumoniae in Taiwan. PLoS ONE. (2014) 9:e96292. doi: 10.1371/journal.pone.0096292

9. Ko W, Paterson D, Sagnimeni A, Hansen D, Von Gottberg A, Mohapatra $\mathrm{S}$, et al. Community-acquired Klebsiella pneumoniae bacteremia: global differences in clinical patterns. Emerging Infect Dis. (2002) 8:160-6. doi: 10.3201/eid0802.010025

10. Fang C, Chuang Y, Shun C, Chang S, Wang J. A novel virulence gene in Klebsiella pneumoniae strains causing primary liver abscess 
and septic metastatic complications. J Exp Med. (2004) 199:697-705. doi: 10.1084/jem.20030857

11. Pomakova D, Hsiao C, Beanan J, Olson R, Macdonald U, Keynan $\mathrm{Y}$, et al. Clinical and phenotypic differences between classic and hypervirulent Klebsiella pneumonia: an emerging and under-recognized pathogenic variant. Eur J Clin Microbiol Infect Dis. (2012) 31:981-9. doi: 10.1007/s10096-011-1396-6

12. Zhang Y, Sun J, Mi C, Li W, Zhao S, Wang Q, et al. First report of two rapid-onset fatal infections caused by a newly emerging hypervirulent $K$. Pneumonia ST86 strain of serotype K2 in China. Front Microbiol. (2015) 6:721. doi: 10.3389/fmicb.2015.00721

13. Qi Y, Wei Z, Ji S, Du X, Shen P, Yu Y. ST11, the dominant clone of KPCproducing Klebsiella pneumoniae in China. J Antimicrob Chemother. (2011) 66:307-12. doi: 10.1093/jac/dkq431

14. Pitout J, Nordmann P, Poirel L. Carbapenemase-producing Klebsiella pneumoniae, a key pathogen set for global nosocomial dominance. Antimicrob Agents Chemother. (2015) 59:5873-84. doi: 10.1128/AAC.01019-15

15. Bi W, Liu H, Dunstan R, Li B, Torres V, Cao J, et al. Extensively drug-resistant causing nosocomial bloodstream infections in China: molecular investigation of antibiotic resistance determinants, informing therapy, and clinical outcomes. Front Microbiol. (2017) 8:1230. doi: 10.3389/fmicb.2017.01230

16. Peirano G, Bradford P, Kazmierczak K, Chen L, Kreiswirth B, Pitout J. Importance of clonal complex 258 and IncF plasmids among a global collection of Klebsiella pneumoniae with. Antimicrob Agents Chemother. (2017) 61. doi: 10.1128/AAC.02610-16

17. Chen L, Mathema B, Pitout J, Deleo F, Kreiswirth B. Epidemic Klebsiella pneumoniae ST258 is a hybrid strain. MBio. (2014) 5: e01314-e01355. doi: $10.1128 / \mathrm{mBio} .01355-14$

18. D'andrea M, Amisano F, Giani T, Conte V, Ciacci N, Ambretti S, et al. Diversity of capsular polysaccharide gene clusters in Kpc-producing Klebsiella pneumoniae clinical isolates of sequence type 258 involved in the Italian epidemic. PLoS ONE. (2014) 9:e96827. doi: 10.1371/journal.pone.0096827

19. Deleo F, Chen L, Porcella S, Martens C, Kobayashi S, Porter A, et al. Molecular dissection of the evolution of carbapenem-resistant multilocus sequence type 258 Klebsiella pneumoniae. Proc Natl Acad Sci USA. (2014) 111:4988-93. doi: 10.1073/pnas.1321364111

20. Wright M, Perez F, Brinkac L, Jacobs M, Kaye K, Cober E, et al. Population structure of KPC-producing Klebsiella pneumoniae isolates from midwestern U.S. hospitals. Antimicrob Agents Chemother. (2014) 58:4961-5. doi: 10.1128/AAC.00125-14

21. Lee C, Lee J, Park K, Kim Y, Jeong B, Lee S. Global dissemination of carbapenemase-producing Klebsiella pneumoniae: epidemiology, genetic context, treatment options, and detection methods. Front Microbiol. (2016) 7:895. doi: $10.3389 /$ fmicb. 2016.00895

22. Dsouza R, Pinto NA, Hwang I, Younjee H, Cho Y, Kim H, et al. Molecular epidemiology and resistome analysis of multidrug-resistant ST11 Klebsiella pneumoniae strain containing multiple copies of extendedspectrum $\beta$-lactamase genes using whole-genome sequencing. New Microbiol. (2017) 40:38-44. Available online at: http://www.newmicrobiologica.org/ PUB/allegati_pdf/2017/1/38.pdf

23. Dong N, Yang X, Zhang R, Chan E, Chen S. Tracking microevolution events among ST11 carbapenemase-producing hypervirulent Klebsiella pneumoniae outbreak strains. Emerg Microbes Infect. (2018) 7:146. doi: 10.1038/s41426-018-0146-6

24. Gu D, Dong N, Zheng Z, Lin D, Huang M, Wang L, et al. A fatal outbreak of ST11 carbapenem-resistant hypervirulent Klebsiella pneumoniae in a Chinese hospital: a molecular epidemiological study. Lancet Infect Dis. (2018) 18:3746. doi: 10.1016/S1473-3099(17)30489-9

25. Gu D, Lv H, Sun Q, Shu L, Zhang R. Emergence of tet(A) and bla $\mathrm{KPC}-2$ co-carrying plasmid from a ST11 hypervirulent Klebsiella pneumoniae isolate in patient's gut. Int J Antimicrob Agents. (2018) 52:307-8. doi: 10.1016/j.ijantimicag.2018.06.003

26. Yao B, Xiao X, Wang $F$, Zhou L, Zhang $X$, Zhang J. Clinical and molecular characteristics of multi-clone carbapenem-resistant hypervirulent (hypermucoviscous) Klebsiella pneumoniae isolates in a tertiary hospital in Beijing, China. Int J Infect Dis. (2015) 37:107-12. doi: $10.1016 /$ j.ijid.2015.06.023
27. Shankar C, Nabarro L, Devanga Ragupathi N, Muthuirulandi Sethuvel D, Daniel J, Doss C, et al. Draft genome sequences of three hypervirulent carbapenem-resistant Klebsiella pneumoniae isolates from bacteremia. Genome Announc. (2016) 4:e01081-16. doi: 10.1128/genomeA.01081-16

28. Chen L, Kreiswirth B. Convergence of carbapenem-resistance and hypervirulence in Klebsiella pneumoniae. Lancet Infect Dis. (2018) 18:2-3. doi: 10.1016/S1473-3099(17)30517-0

29. Lu M, Tang H, Chiou C, Wang Y, Chiang M, Lai Y. Clonal dissemination of carbapenemase-producing Klebsiella pneumoniae: Two distinct sub-lineages of Sequence Type 11 carrying $b l a_{\mathrm{KPC}-2}$ and bla $a_{\mathrm{OXA}-48}$. Int J Antimicrob Agents. (2018) 52:658-62. doi: 10.1016/j.ijantimicag.2018.04.023

30. Yu F, Lv J, Niu S, Du H, Tang Y, Pitout J, et al. Multiplex PCR analysis for rapid detection of Klebsiella pneumoniae carbapenem-resistant (sequence type 258 [ST258] and ST11) and hypervirulent (ST23, ST65, ST86, and ST375) strains. J Clin Microbiol. (2018) 56:e00731. doi: 10.1128/JCM.00731-18

31. Liu Y, Li B, Zhang Y, Zhang W, Shen H, Li H, et al. Clinical and molecular characteristics of emerging hypervirulent Klebsiella pneumoniae bloodstream infections in mainland China. Antimicrob Agents Chemother. (2014) 58:537985. doi: 10.1128/AAC.02523-14

32. Lin $\mathrm{W}$, Wang $\mathrm{M}$, Tseng $\mathrm{C}, \mathrm{Ko} \mathrm{W}, \mathrm{Wu} \mathrm{A}$, Zheng $\mathrm{P}$, et al. Clinical and microbiological characteristics of Klebsiella pneumoniae isolates causing community-acquired urinary tract infections. Infection. (2010) 38:459-64. doi: 10.1007/s15010-010-0049-5

33. Romero L, López L, Martínez-Martínez L, Guerra B, Hernández J, Pascual A. Characterization of the first CTX-M-14-producing Salmonella enterica serotype Enteritidis isolate. J Antimicrob Chemother. (2004) 53:1113-4. doi: $10.1093 / \mathrm{jac} / \mathrm{dkh} 246$

34. Tenover F, Arbeit R, Goering R, Mickelsen P, Murray B, Persing D, et al. Interpreting chromosomal DNA restriction patterns produced by pulsedfield gel electrophoresis: criteria for bacterial strain typing. J Clin Microbiol. (1995) 33:2233-9.

35. Insua J, Llobet E, Moranta D, Pérez-Gutiérrez C, Tomás A, Garmendia J, et al. Modeling Klebsiella pneumoniae pathogenesis by infection of the wax moth Galleria mellonella. Infect Immun. (2013) 81:3552-65. doi: 10.1128/IAI.00391-13

36. Manohar P, Nachimuthu R, Lopes B. The therapeutic potential of bacteriophages targeting gram-negative bacteria using Galleria mellonella infection model. BMC Microbiol. (2018) 18:97. doi: $10.1186 / \mathrm{s} 12866-018-1234-4$

37. Shi Q, Lan P, Huang D, Hua X, Jiang Y, Zhou J, et al. Diversity of virulence level phenotype of hypervirulent Klebsiella pneumoniae from different sequence type lineage. BMC Microbiol. (2018) 18:94. doi: 10.1186/s12866-018-1236-2

38. Zhan L, Wang S, Guo Y, Jin Y, Duan J, Hao Z, et al. Outbreak by hypermucoviscous Klebsiella pneumoniae ST11 isolates with carbapenem resistance in a tertiary hospital in China. Front Cell Infect Microbiol. (2017) 7:182. doi: $10.3389 /$ fcimb.2017.00182

39. Hsu C, Lin T, Chen Y, Chou H, Wang J. The role of Klebsiella pneumoniae rmpA in capsular polysaccharide synthesis and virulence revisited. Microbiology. (2011) 157:3446-57. doi: 10.1099/mic.0.050336-0

40. Lin $\mathrm{T}$, Yang $\mathrm{F}$, Yang $\mathrm{A}$, Peng $\mathrm{H}$, Li $\mathrm{T}$, Tsai $\mathrm{M}$, et al. Amino acid substitutions of MagA in Klebsiella pneumoniae affect the biosynthesis of the capsular polysaccharide. PLOS ONE. (2012) 7:e46783. doi: 10.1371/journal.pone.0046783

41. Zheng J, Lin Z, Chen C, Chen Z, Lin F, Wu Y, et al. Biofilm formation in bacteremia strains was found to be associated with $\mathrm{CC} 23$ and the presence of wcaG. Front Cell Infect Microbiol. (2018) 8:21. doi: 10.3389/fcimb.2018.00021

42. Haldorsen B, Simonsen G, Sundsfjord A, Samuelsen O. Increased prevalence of aminoglycoside resistance in clinical isolates of Escherichia coli and Klebsiella spp. in Norway is associated with the acquisition of AAC(3)-II and AAC(6')-Ib. Diagn Microbiol Infect Dis. (2014) 78:66-9. doi: 10.1016/j.diagmicrobio.2013.10.001

43. Sanchez-Lopez J, Garcia-Caballero A, Navarro-San Francisco C, Quereda C, Ruiz-Garbajosa P, Navas E, et al. Hypermucoviscous Klebsiella pneumoniae: a challenge in community acquired infection. IDCases. (2019) 17:e00547. doi: 10.1016/j.idcr.2019.e00547

44. Catalán-Nájera J, Garza-Ramos U, Barrios-Camacho H. Hypervirulence and hypermucoviscosity: two different but complementary 
Klebsiella spp. phenotypes? Virulence. (2017) 8:1111-23. doi: 10.1080/21505594.2017.1317412

45. Schroll C, Barken K, Krogfelt K, Struve C. Role of type 1 and type 3 fimbriae in Klebsiella pneumoniae biofilm formation. BMC Microbiol. (2010) 10:179. doi: 10.1186/1471-2180-10-179

46. Wilksch J, Yang J, Clements A, Gabbe J, Short K, Cao H, et al. MrkH, a novel cdi-GMP-dependent transcriptional activator, controls Klebsiella pneumoniae biofilm formation by regulating type 3 fimbriae expression. PLoS Pathog. (2011) 7:e1002204. doi: 10.1371/journal.ppat.1002204

47. Wasfi R, Elkhatib WF, Ashour HM. Molecular typing and virulence analysis of multidrug resistant Klebsiella pneumoniae clinical isolates recovered from Egyptian hospitals. Sci Rep. (2016) 6:38929. doi: 10.1038/srep38929

48. Chou H, Lee C, Ma L, Fang C, Chang S, Wang J. Isolation of a chromosomal region of Klebsiella pneumoniae associated with allantoin metabolism and liver infection. Infect Immun. (2004) 72:3783-92. doi: 10.1128/IAI.72.7.3783-3792.2004

49. Maroncle N, Rich C, Forestier C. The role of Klebsiella pneumoniae urease in intestinal colonization and resistance to gastrointestinal stress. Res Microbiol. (2006) 157:184-93. doi: 10.1016/j.resmic.2005.06.006

50. Harada S, Aoki K, Ishii Y, Ohno Y, Nakamura A, Komatsu M, et al. Emergence of IMP-producing hypervirulent Klebsiella pneumoniae carrying a pLVPK-like virulence plasmid. Int J Antimicrob Agents. (2019) 53:873-5. doi: 10.1016/j.ijantimicag.2019.05.007

51. Liu YY, Wang Y, Walsh TR, Yi LX, Zhang R, Spencer J, et al. Emergence of plasmid-mediated colistin resistance mechanism MCR-1 in animals and human beings in China: a microbiological and molecular biological study. Lancet Infect Dis. (2016) 16:161-8. doi: 10.1016/S1473-3099(15)00424-7

52. Cao L, Li X, Xu Y, Shen J. Prevalence and molecular characteristics of $m c r-1$ colistin resistance in Escherichia coli: isolates of clinical infection from a Chinese University Hospital. Infect Drug Resist. (2018) 11:1597-603. doi: 10.2147/IDR.S166726

53. Olaitan AO, Morand S, Rolain JM. Mechanisms of polymyxin resistance: acquired and intrinsic resistance in bacteria. Front Microbiol. (2014) 5:643. doi: $10.3389 /$ fmicb. 2014.00643

54. Feldman N, Adler A, Molshatzki N, Navon-Venezia S, Khabra E, Cohen D, et al. Gastrointestinal colonization by KPC-producing Klebsiella pneumoniae following hospital discharge: duration of carriage and risk factors for persistent carriage. Clin Microbiol Infect. (2013) 19:E190-6. doi: 10.1111/1469-0691.12099

55. Lübbert, C., Lippmann, N., Busch, T., Kaisers, U., Ducomble, T., Eckmanns, T., et al. (2014). Long-term carriage of Klebsiella pneumoniae carbapenemase2-producing K.pneumoniae after a large single-center outbreak in Germany. Am J Infect Control. 42, 376-380. doi: 10.1016/j.ajic.2013.12.001

56. Conlan S, Park M, Deming C, Thomas P, Young A, Coleman H, et al. Plasmid dynamics in KPC-positive Klebsiella pneumoniae during long-term patient colonization. MBio. (2016) 7:e00742-16. doi: 10.1128/mBio.00742-16

57. Yang J, Ye L, Guo L, Zhao Q, Chen R, Luo Y, et al. A nosocomial outbreak of KPC-2-producing Klebsiella pneumoniae in a Chinese hospital: dissemination of ST11 and emergence of ST37, ST392 and ST395. Clin Microbiol Infect. (2013) 19:E509-515. doi: 10.1111/1469-0691.12275

58. Zhou T, Zhang Y, Li M, Yu X, Sun Y, Xu J. An outbreak of infections caused by extensively drug-resistant Klebsiella pneumoniae strains during a short period of time in a Chinese teaching hospital: epidemiology study and molecular characteristics. Diagn Microbiol Infect Dis. (2015) 82:240-4. doi: 10.1016/j.diagmicrobio.2015.03.017
59. Hu L, Liu Y, Deng L, Zhong Q, Hang Y, Wang Z, et al. Outbreak by ventilatorassociated ST11 K. pneumoniae with co-production of CTX-M-24 and KPC-2 in a SICU of a Tertiary Teaching Hospital in Central China. Front Microbiol. (2016) 7:1190. doi: 10.3389/fmicb.2016.01190

60. Sui W, Zhou H, Du P, Wang L, Qin T, Wang M, et al. Whole genome sequence revealed the fine transmission map of carbapenem-resistant Klebsiella pneumonia isolates within a nosocomial outbreak. Antimicrob Resist Infect Control. (2018) 7:70. doi: 10.1186/s13756-018-0363-8

61. Liu Z, Gu Y, Li X, Liu Y, Ye Y, Guan S, et al. Identification and characterization of NDM-1-producing hypervirulent (Hypermucoviscous) Klebsiella pneumoniae in China. Ann Lab Med. (2019) 39:167-75. doi: 10.3343/alm.2019.39.2.167

62. Van Dorp L, Wang Q, Shaw LP, Acman M, Brynildsrud OB, Eldholm $\mathrm{V}$, et al. Rapid phenotypic evolution in multidrug-resistant Klebsiella pneumoniae hospital outbreak strains. Microb Genom. (2019) 5:e000263. doi: 10.1099/mgen.0.000263

63. Gilchrist CA, Turner SD, Riley MF, Petri WAJr, Hewlett EL. Whole-genome sequencing in outbreak analysis. Clin Microbiol Rev. (2015) 28:541-63. doi: 10.1128/CMR.00075-13

64. Araujo BF, Ferreira ML, Campos PA, Royer S, Goncalves IR, Da Fonseca Batistao DW, et al. Hypervirulence and biofilm production in KPC-2producing Klebsiella pneumoniae CG258 isolated in Brazil. J Med Microbiol. (2018) 67:523-8. doi: 10.1099/jmm.0.000711

65. Li W, Sun G, Yu Y, Li N, Chen M, Jin R, et al. Increasing occurrence of antimicrobial-resistant hypervirulent (hypermucoviscous) Klebsiella pneumoniae isolates in China. Clin Infect Dis. (2014) 58:225-32. doi: 10.1093/cid/cit675

66. Bialek-Davenet S, Criscuolo A, Ailloud F, Passet V, Jones L, DelannoyVieillard A-S, et al. Genomic definition of hypervirulent and multidrugresistant Klebsiella pneumoniae clonal groups. Emerg Infect Dis. (2014) 20:1812. doi: 10.3201/eid2011.140206

67. Ramarao N, Nielsen-Leroux C, Lereclus D. The insect Galleria mellonella as a powerful infection model to investigate bacterial pathogenesis. $J$ Vis Exp. (2012) 11:e4392. doi: 10.3791/4392

68. Mclaughlin M, Advincula M, Malczynski M, Barajas G, Qi C, Scheetz M. Quantifying the clinical virulence of Klebsiella pneumoniae producing carbapenemase Klebsiella pneumoniae with a Galleria mellonella model and a pilot study to translate to patient outcomes. BMC Infect Dis. (2014) 14:31. doi: 10.1186/1471-2334-14-31

69. Browne N, Heelan M, Kavanagh K. An analysis of the structural and functional similarities of insect hemocytes and mammalian phagocytes. Virulence. (2013) 4:597-603. doi: 10.4161/viru. 25906

Conflict of Interest Statement: The authors declare that the research was conducted in the absence of any commercial or financial relationships that could be construed as a potential conflict of interest.

Copyright (๑) 2019 Zhao, Zhang, Torres, Liu, Rocker, Zhang, Wang, Chen, Bi, Lin, Strugnell, Zhang, Lithgow, Zhou and Cao. This is an open-access article distributed under the terms of the Creative Commons Attribution License (CC BY). The use, distribution or reproduction in other forums is permitted, provided the original author(s) and the copyright owner(s) are credited and that the original publication in this journal is cited, in accordance with accepted academic practice. No use, distribution or reproduction is permitted which does not comply with these terms. 